\title{
Does trade always harm the global environment? A case for positive interaction
}

\author{
By Savas Alpay \\ Department of Economics, Bilkent University, Bilkent, 06533 Ankara, Turkey
}

\begin{abstract}
We demonstrate that there are links between international trade and environmental control, heretofore unappreciated, which might substantially alter the efficacy of various governmental policies to control pollution. One concern about national environmental policies is that, whereas the benefits of certain types of abatement might be international or even worldwide, the costs will be borne strictly by the consumers and firms of the country which institutes the policy. As a result, for those types of pollution which are global (such as greenhouse gases) there will be too little pollution abatement. Our first result is that this pessimistic conclusion may be unwarranted. In a $2 \times 2$ Ricardian model, we also show cases where the non-cooperative contribution of countries to global environmental protection, contrary to the conventional results, exceeds that of the cooperative one due to associated changes in the terms of trade. Thus, international trade is not always a threat to global environment.
\end{abstract}

\section{Introduction}

The global environment is subject to two adverse externalities. Transnational environmental degradation, a type of negative externality, imposes certain costs not only on the originating country but also on the other countries (ozone-layer depletion is a good example of this). On the other hand, policies aimed to tackle transnational environmental degradation, involve a positive externality from which all countries benefit regardless of their taking action. As a result, countries leave it to others to take pro-environmental actions, and this may result in under-production (if not zero) of the environmental good. ${ }^{1}$ Thus, as in the public good case, global environmental protection is subject to free-riding.

Barrett (1994a), Heal (1994), and Carraro and Siniscalco (1993) study international agreements to protect the environment and free-riding ${ }^{2}$ in the context mentioned above. Barrett (1994a) shows that self-enforcing international environ-

\footnotetext{
${ }^{1}$ In this paper, environmental protection, pro-environmental policy and other similar terms should be considered, in economic terms, as the production of environmental good.

${ }^{2}$ In an earlier literature, there has been put some doubt on the importance and the existence of the free rider problem. Explicitly, we can cite Bohm (1972), Sweeney (1973), and Smith (1979), who designed experiments which resulted in negligible free-riding when the agents had strong incentives to free ride. Nevertheless, Kim and Walker (1984) suggested that there were some invalidating factors in these previous experiments and by removing these factors in their experiment, they found out that, in fact, free-riding was substantial.
} 
mental agreements (IEAs) may not be able to improve substantially over the noncooperative outcome. There are cases when self-enforcing IEAs can sustain a large number of countries, but this requires that the difference in net benefits between the non-cooperative and full-cooperative outcome is very small. Heal (1994) studies the formation of emissions abatement coalitions. In a game theoretical model, Heal shows that the selection of positive abatement levels by other countries may shift a particular country's best choice of abatement from zero to a positive number if the benefit and cost functions of identical countries are not separable across abatement levels (reinforcement effects), of if there are fixed costs associated with abatement programs; thus, free-riding need not always be the best action. Carraro and Siniscalco (1993) analyze the profitability and stability (no free-riding) of international environmental agreements in the presence of trans-boundary pollution. They show that the agreements tend to be realized among a fraction of negotiating countries and that the number of signatories can be increased through self-financed transfers; however, any expansion of a coalition requires some form of commitment, which is a strong assumption.

Other studies carry out the analysis in an incomplete information framework. Petrakis and Xepapadeas (1996) analyze global or partial stable coalitions among two groups of countries, which differ from each other in terms of environmental consciousness, through self-financed transfers. They also demonstrate a mechanism which detects free-riding (or cheating) even when the individual emissions cannot be observed by other countries. Bac (1996) studies free-riding incentives on common environmental resources in an incomplete information repeated game involving two countries. His results include the case where one country bears the burden of abating forever, an extreme example of free-riding.

Earlier, Demsetz (1967) presented some very sharp views on this issue, shared by many others. He states that users of a communally owned resource will fail to come to an agreement on managing the use of the resource even though it is in the interest of all users to cooperate and reduce the amount of the resource they use. This is so because, even if this superior situation is attained, every user will attain even higher returns by free-riding on the cooperative behaviour of the others. The only solution according to Demsetz (1967) is some kind of state intervention. Taking these views of Demsetz (1967) as his starting point, Barrett (1990) states that the theoretical arguments for supposing that cooperation will not develop are compelling, but they cannot be complete as cooperation (in some form) does take place and is codified in international agreements (whether effective or not). $\mathrm{He}$ then goes on to explore the reasons why international cooperation might develop.

In this paper, we will add an important result to this literature. Global environmental protection should not and cannot be studied by itself, in a partial equilibrium framework. Given the connections among countries in the international markets for private goods, it is of interest to determine the impact of international trade on incentives to free ride on global environmental protection. One of the first studies along these lines is done by Barrett (1997). His paper develops a stylized 
model of international trade and environmental cooperation in which the threat to impose trade sanctions emerges as an equilibrium. His model shows that there will not be any trade restriction in equilibrium; however, if the threat to impose trade sanctions is prohibited, for example due to GATT regulations, international cooperation could only sustain a collectively inferior outcome.

Our paper will highlight one other aspect of the interactions between trade and environmental protection. There are economic links between global environmental protection and international trade and they need to be examined in a general equilibrium framework. This is so because production of an environmental good will be accompanied by drawing resources from the private sector of the economy which engages in the production of goods and services that are internationally traded. Thus, in addition to environmental benefits, there will also be terms of trade and income effects which must be included in the evaluation of environmental policies by the governments. As a policy implication, we can state that the assessment of government policies on global environmental protection in a partial equilibrium framework (by ignoring the possible interactions mentioned above) may very well be misleading.

It turns out that incorporation of these connections to the model generates very interesting results. First of all, we show that non-cooperative Nash equilibrium may very well result in no free-riding under reasonable assumptions. Self-financed transfers to avoid free-riding as suggested by the earlier papers mentioned above are not always required. Therefore, we reinforce Heal's (1994) non-conventional findings above, in a general equilibrium model which also incorporates the important trade connections among countries and discloses the impact of trade on these results. Secondly, and maybe more importantly, contributions to global environmental protection by each country can be higher in the non-cooperative case than in the cooperative case, contrary to the conventional results. So, the underproduction of public goods in non-cooperative settings in closed economies does not easily extend to open economy case. Why are these so? The main driving force is the changes in the terms of trade of countries associated with contributions to global environmental protection. With the assumption that trade patterns remain the same, the benefits to employing resources in the environmental sector become larger and thus more labor becomes employed in that sector as a result of indirect trade effects. To reinforce this conclusion, we also consider the Nash equilibrium contribution of countries to global environment when terms of trade changes are ignored, and show that this time non-cooperative contribution will be less than the contribution corresponding to cooperative solution, which is the conventional result.

A similar exploration has been done in the literature of localized environmental problems. Barrett (1994b) considers the possibilities where the environmental agency tightens environmental policies in order to maintain competitiveness through terms of trade gains instead of (or in addition to) competitive loosening of policy. He finds that, for oligopolistic industries with perfectly competitive 
foreign competition, strategically optimal policy level (for localized environmental damage) can be either greater or lower than the social optimal.

In an empirical study, Piggott et al. (1992) argue that 'the incentives to either participate or not to participate in any international initiatives to reduce carbon emissions reflect much more than free-riding on the benefit side'. A cut in energy use by one country confers benefits to others as well, but the overall welfare assessment of such an action also depends on production substitution across countries, and on terms of trade effects involving energy products, energy-intensive and other products. They show that terms of trade effects associated with reductions in emissions are potentially important, and the numerical values for percentage changes in terms of trade are very high. In our paper, we will now add theoretical arguments that support the Piggott et al. findings. We will concentrate on general global environmental protection (not specifically on carbon reduction schemes as in Piggott et al., 1992) and its connections with international trade through terms of trade changes.

Not all studies agree that the interaction between international trade and the global environment is positive. Chilchilnisky (1994) demonstrates that if private property rights are not established, trade will increase the resource extraction in the developing countries, and thus, may be detrimental to global environment. Daly (1993) looks at how international trade impacts (global) pollution and he concludes that trade increases pollution. Such results put doubt on the importance of trade liberalization. This paper presents a counter evidence on this issue and thus becomes more valuable by showing a case for international trade from an environmental perspective. Hence, overall evaluation of trade and environment relations must take these different perspectives into account.

To sum up, there are links between international trade and environmental control, heretofore unappreciated, which might substantially alter the efficacy of various governmental policies to control pollution. The impact of proenvironmental policy on the terms of trade can outweigh the environmental gains and thus free-riding may not be a welfare-improving choice. As a policy implication, this requires that the governments consider all aspects of their environmental policies, at least the ones that pertain to international trade. Chilchilnisky (1994) states that 'the international market transmits and enlarges the externalities of the global commons. No policy that ignores this connection can work'. We agree with her results; however, in this paper, we show that international market can also transmit and enlarge global environmental protection across countries by reducing free-riding incentives. Thus, trade is not always a threat to global environment.

The organization of the paper is as follows: in the next section we introduce our 2 by 2 model. Then, we analyze production of environmental good, freeriding possibilities, Nash equilibrium of the non-cooperative environmental game, and the corresponding cooperative solution. We finish with concluding remarks. 


\section{Model}

We will take up a two-country two-good Ricardian model. Each country consumes two goods, $X$ and $Y$. There is only one factor of production, labor. Total labor endowment in home country is $\bar{L}$, and the one in foreign country is $\bar{L}^{*}$. Consumers demand environmental quality along with private consumption goods, and demand for environmental quality is represented by the demand for environmental good, F. Our model will allow for substitution between private goods and environmental quality. The substitution effect is usually ignored in the literature with the exception of a few studies. For example, Smith and Espinosa (1996) point out the importance of this link by stating that 'pollution itself may alter the composition of goods demanded'. In our case, the substitution effect will show itself through the impact of environmental protection on the income, which will be shown explicitly below. Higher demand for environmental quality will attract more resources to environmental sector, and the compensation for these resources will come from taxes paid by consumers; therefore, demand for private goods will decline. Moreover, the environmental agency (or government) chooses the level of environmental protection in a way that maximizes the welfare of the consumers; therefore, the parameters that affect the demand for private goods will also have impact on the level of environmental protection.

\subsection{Production}

Production functions are assumed to be linear in labor input. Unit labor requirements are $h_{x}, h_{y}$, and $h_{f}$ for goods $X, Y$, and $F$, respectively, in home country and $f_{x}, f_{y}$, and $f_{f}$ in foreign country. We assume that full employment of labor prevails in both countries, so $\bar{L}=L_{X}+L_{Y}+L_{F}$ and $\bar{L}^{*}=L_{X}^{*}+L_{Y}^{*}+L_{F}^{*}$. Throughout our analysis we will assume that these unit labor requirements are such that home country specializes in the production of good $X$ and foreign country in the production of $Y$. A couple of remarks on the environmental good $F$ are in order.

In production terms, $F$ can be interpreted as a public good which represents the cumulative contribution of a country to environmental protection. More explicitly, we formalize the total amount of resources devoted to environmental protection through the production of $F$. An important nature of the environmental good $F$ is that it not only benefits the country which produces it, but also benefits the other country. Therefore, free-riding will be an important concern in the production of environmental good. In a closed economy, Nash equilibrium production of a good subject to free-riding will be lower than the level corresponding to the cooperative solution. Now, the question is whether this result will still be valid in an open economy.

Under the assumed pattern of trade, the incomes of home and foreign country will be $I=P_{x}\left(1 / h_{x}\right)\left(\bar{L}-L_{F}\right)$ and $I^{*}=P_{y}\left(1 / h_{y}\right)\left(\bar{L}^{*}-L_{F}^{*}\right)$, respectively. We note that production of $F$ is financed by taxation. 


\subsection{Demand}

We assume that the representative consumer ${ }^{3}$ has the following Cobb-Douglas utility function

$$
U\left(X, Y, F, F^{*}\right)=X^{\alpha} Y^{\gamma}\left(1+F+\lambda F^{*}\right)^{\beta}
$$

As usual, $\alpha$ and $\gamma$ represent, the share of $X$ and $Y$ in total expenditure, respectively, and thus the value of these two goods to the consumer; $\beta$ plays the same role for environmental quality. Since global environmental protection by any country contributes to the welfare of both countries, utility function includes both $F$ and $F^{*}$; however, these contributions are asymmetric as represented by $\lambda ; \lambda$ measures both the degree of free-riding, and the degree of substitution between environmental protection of home country and that of foreign country. Consumer maximizes this utility function subject to the budget constraint, which is $P_{x} X+P_{y} Y=I$.

\section{Free-riding and Nash equilibrium}

In this section, we will study the Nash equilibrium of the game where both countries decide whether they should produce environmental good $F$ or not, and if affirmative, how-much they should produce. It is of interest to determine any possible impact of the trade connections between countries on this choice of contribution to environmental protection. Since a contribution to global environmental protection requires the transfer of some of the resources from the private sector to the environmental sector, the interaction between trade and the environment can be seen by considering the changes in the terms of trade associated with this resource shift. Towards that end, we will determine the Nash equilibrium contributions of the countries when terms of trade changes resulting from the production of $F$ are (i) incorporated and (ii) ignored. We can, then, make an assessment on whether trade connections may reduce free-riding incentives by comparing the level of contributions in each case. Having done these, the natural next step is to show the cooperative solution for this same problem and compare the results with those in the non-cooperative case. With this comparison, we will be able to assess whether the underproduction of the public goods in the closed economy will extend to the open economy case (when there are trade connections). After this overview, we can now see the details.

One may separate the overall process into two stages. In the first stage, the representative consumer maximizes his/her utility over the private goods for a given level of domestic and foreign environmental protection, $F$ and $F^{*}$, respectively. In the next stage, domestic environmental agency chooses the amount of $F$ such that the utility of the representative consumer as computed in the first stage is maximized. The consumer's problem will be as follows

\footnotetext{
${ }^{3}$ For simplicity, we will assume that there is only one consumer in both home and foreign country, or many consumers with identical utility functions so that we can use a social utility function which is equal to the sum of the individual utility functions, as in Smith and Espinosa (1996).
} 


$$
\max _{X, Y} U\left(X, Y, F, F^{*}\right)=X^{\alpha} Y^{\gamma}\left(1+F+\lambda F^{*}\right)^{\beta}
$$

subject to the budget constraint, $P_{x} X+P_{y} Y=I$, where $I=P_{x}\left(\bar{L}-L_{F}\right) / h_{x}$. Allocation of some of the resources to environmental protection $\left(L_{F}\right)$ will affect private income as reflected in the budget equation.

The resulting demand functions are

$$
\begin{aligned}
& X=\frac{\alpha}{(\alpha+\gamma)} \frac{I}{P_{x}}=\frac{\alpha}{(\alpha+\gamma)} \frac{\left(\bar{L}-L_{F}\right)}{h_{x}} \\
& Y=\frac{\gamma}{(\alpha+\gamma)} \frac{I}{P_{y}}=\frac{\gamma}{(\alpha+\gamma)} \frac{P_{x}}{P_{y}} \frac{\left(\bar{L}-L_{F}\right)}{h_{x}}
\end{aligned}
$$

Since the foreign country specializes in the production of $Y$, the demand functions for its representative consumer will be

$$
\begin{aligned}
X^{*} & =\frac{\alpha}{(\alpha+\gamma)} \frac{P_{y}}{P_{x}} \frac{\left(\bar{L}^{*}-L_{F}^{*}\right)}{f_{y}} \\
Y^{*} & =\frac{\gamma}{(\alpha+\gamma)} \frac{\left(\bar{L}^{*}-L_{F}^{*}\right)}{f_{y}}
\end{aligned}
$$

Trade balance requires that export receipts equal import payments; since the home country imports good $Y$ and exports good $X$, the following relation must hold $^{4}$

$$
P_{y} Y=P_{x} X^{*}
$$

Replacing demand functions, we obtain the terms of trade as

$$
\frac{P_{x}}{P_{y}}=\frac{\alpha}{\gamma} \frac{h_{x}}{f_{y}} \frac{\left(\bar{L}^{*}-L_{F}^{*}\right)}{\left(\bar{L}-L_{F}\right)}
$$

Given the optimal choices of $X$ and $Y$, and the trade balance equation, the representative consumer's maximum utility can be expressed as a function of $L_{F}$ and $L_{F}^{*}$ as

$$
U\left(X, Y, F, F^{*}\right)=\frac{\alpha^{\alpha+\gamma}}{(\alpha+\gamma)^{\alpha+\gamma}} \frac{1}{h_{x}^{\alpha} f_{y}^{\gamma}}\left(\bar{L}^{*}-L_{F}^{*}\right)^{\gamma}\left(\bar{L}-L_{F}\right)^{\alpha}\left(1+\frac{L_{F}}{h_{f}}+\lambda \frac{L_{F}^{*}}{f_{f}}\right)^{\beta}
$$

Now, we are at the second stage. The environmental agency chooses $L_{F}$ such that the utility of the consumer expressed in (8) reaches a maximum. The problem of the agency is

$$
\max _{L_{F}} \frac{\alpha^{\alpha+\gamma}}{(\alpha+\gamma)^{\alpha+\gamma}} \frac{1}{h_{x}^{\alpha} f_{y}^{\gamma}}\left(\bar{L}^{*}-L_{F}^{*}\right)^{\gamma}\left(\bar{L}-L_{F}\right)^{\alpha}\left(1+\frac{L_{F}}{h_{f}}+\lambda \frac{L_{F}^{*}}{f_{f}}\right)^{\beta}
$$

From the solution of first order conditions, we obtain

\footnotetext{
${ }^{4}$ Note that trade balance together with budget constraints results in the equality of demand and supply worldwide, i.e., $X+X^{*}=X_{S}$, and $Y+Y^{*}=Y_{S}$, where $X_{S}$ and $Y_{S}$ represent supply of goods.
} 


$$
L_{F}=\frac{\beta}{\alpha+\beta} \bar{L}-\frac{\alpha}{\alpha+\beta} h_{f}-\frac{\alpha \lambda h_{f}}{(\alpha+\beta) f_{f}} L_{F}^{*}
$$

Equation (9) is the reaction function of home country with respect to $L_{F}^{*}$ of foreign country. A similar analysis for foreign country results in the following reaction function for foreign country with respect to $L_{F}$ of home country

$$
L_{F}^{*}=\frac{\beta}{\gamma+\beta} \bar{L}^{*}-\frac{\gamma}{\gamma+\beta} f_{f}-\frac{\gamma \lambda f_{f}}{(\gamma+\beta) h_{f}} L_{F}
$$

To determine the Nash equilibrium of this game, we first calculate the welfare levels associated with each of the four possible outcomes, by using the optimal contributions above in (9) and (10) when they choose to contribute:

Case I Both countries contribute

$$
\begin{gathered}
U\left(X, Y, F, F^{*}\right)=\frac{\alpha^{\alpha+\gamma}}{(\alpha+\gamma)^{\alpha+\gamma}} \frac{1}{h_{x}^{\alpha} f_{y}^{\gamma}}\left(\bar{L}^{*}-L_{F}^{*}\right)^{\gamma}\left(\bar{L}-L_{F}\right)^{\alpha}\left(1+\frac{L_{F}}{h_{f}}+\lambda \frac{L_{F}^{*}}{f_{f}}\right)^{\beta} \\
U^{*}\left(X^{*}, Y^{*}, F, F^{*}\right)=\frac{\gamma^{\alpha+\gamma}}{(\alpha+\gamma)^{\alpha+\gamma}} \frac{1}{h_{x}^{\alpha} f_{y}^{\gamma}}\left(\bar{L}^{*}-L_{F}^{*}\right)^{\gamma}\left(\bar{L}-L_{F}\right)^{\alpha}\left(1+\frac{L_{F}^{*}}{f_{f}}+\lambda \frac{L_{F}}{h_{f}}\right)^{\beta}
\end{gathered}
$$

Case II Neither country contributes

$$
\begin{gathered}
U\left(X, Y, F, F^{*}\right)=\frac{\alpha^{\alpha+\gamma}}{(\alpha+\gamma)^{\alpha+\gamma}} \frac{1}{h_{x}^{\alpha} f_{y}^{\gamma}} \bar{L}^{* \gamma} \bar{L}^{\alpha} \\
U^{*}\left(X^{*}, Y^{*}, F, F^{*}\right)=\frac{\gamma^{\alpha+\gamma}}{(\alpha+\gamma)^{\alpha+\gamma}} \frac{1}{h_{x}^{\alpha} f_{y}^{\gamma}} \bar{L}^{* \gamma} \bar{L}^{\alpha}
\end{gathered}
$$

Case III The home country contributes, the foreign country does not

$$
\begin{gathered}
U\left(X, Y, F, F^{*}\right)=\frac{\alpha^{\alpha+\gamma}}{(\alpha+\gamma)^{\alpha+\gamma}} \frac{1}{h_{x}^{\alpha} f_{y}^{\gamma}} \bar{L}^{* \gamma}\left(\bar{L}-L_{F}\right)^{\alpha}\left(1+\frac{L_{F}}{h_{f}}\right)^{\beta} \\
U^{*}\left(X^{*}, Y^{*}, F, F^{*}\right)=\frac{\gamma^{\alpha+\gamma}}{(\alpha+\gamma)^{\alpha+\gamma}} \frac{1}{h_{x}^{\alpha} f_{y}^{\gamma}} \bar{L}^{* \gamma}\left(\bar{L}-L_{F}\right)^{\alpha}\left(1+\lambda \frac{L_{F}}{h_{f}}\right)^{\beta}
\end{gathered}
$$

Case IV The foreign country contributes, the home country does not

$$
\begin{gathered}
U\left(X, Y, F, F^{*}\right)=\frac{\alpha^{\alpha+\gamma}}{(\alpha+\gamma)^{\alpha+\gamma}} \frac{1}{h_{x}^{\alpha} f_{y}^{\gamma}}\left(\bar{L}^{*}-L_{F}^{*}\right)^{\gamma} \bar{L}^{\alpha}\left(1+\lambda \frac{L_{F}^{*}}{f_{f}}\right)^{\beta} \\
U^{*}\left(X^{*}, Y^{*}, F, F^{*}\right)=\frac{\gamma^{\alpha+\gamma}}{(\alpha+\gamma)^{\alpha+\gamma}} \frac{1}{h_{x}^{\alpha} f_{y}^{\gamma}}\left(\bar{L}^{*}-L_{F}^{*}\right)^{\gamma} \bar{L}^{\alpha}\left(1+\frac{L_{F}^{*}}{f_{f}}\right)^{\beta}
\end{gathered}
$$

We need to make some simplifying assumptions to be able to track down the Nash equilibrium of this game. One interesting specification is the case of symmetric countries, a very common assumption in this literature. 
Definition The countries are symmetric if $\bar{L}=\bar{L}^{*}, h_{f}=f_{f}$, and $\alpha=\beta=\gamma$. Then, our first result is:

Theorem 1 If the countries are symmetric, and if the following two conditions (i) $\left((1+\lambda) /(2+\lambda)-\left(\lambda / 2 h_{f}\right)\right) \bar{L}>(2-\lambda) / 2-(1 /(2+\lambda)) h_{f}$, (ii) $h_{f}<(1+\lambda) /(2+\lambda)$ are satisfied, then the Nash equilibrium of the game described above does not support free-riding, i.e., both countries choose to contribute to global protection.

Proof of Theorem 1 is given in the Appendix. Note that the conditions of this theorem are not necessary conditions; there may be many other cases, in which these conditions will not be satisfied, but the result of Theorem 1 will still hold.

A common view in the literature of global environmental issues is that cooperation for the protection of global environment is very difficult to obtain, and even if it is realized, every country will earn higher benefits by free-riding on the contribution of other countries (see Demsetz, 1967, for example). This view cannot always be true. Our last theorem shows that due to interactions in international goods markets, free-riding is not always the best policy for the countries. Even in a non-cooperative setting, contribution to global protection brings a higher return for both countries under some conditions. Hence, the reluctance to undertake pro-environmental policies due to free-riding problem is not grounded solidly in an open economy. Let us try to understand the reasons behind this unconventional result.

\subsection{Terms of trade effects}

When both countries start allocating some of their resources in the production of environmental good, the resources available for production in the private sector will decline. As a result, such environmental policies will have terms of trade effects, the magnitude of which should be a function of the resources transferred to environmental sector. This relationship is shown in eq. (7) above.

In the public goods literature, it is known that the agents would like to free ride and the production of the public good will be sub-optimal. This is a conventional result in a closed economy. When the analog of this public good problem in an open economy is developed, one needs to consider the trade related side effects. The obvious side effect is the changes in the terms of trade generated by the production of the public good. Let us state this more formally:

Lemma In an open economy, along with the positive environmental externality, the production of the environmental good also generates a negative externality on the free-riding country and a positive externality on the active country through terms of trade changes.

Proof First we note that the welfare of home country is positively related to the terms of trade, $P_{x} / P_{y}$, which can be seen by considering eqs (1) and (3) together. A similar consideration will show that the utility function of the representative consumer in foreign country is negatively related to the same ratio. The relationship 
between environmental good production (which is a function of $L_{F}$ ), and the terms of trade is given in eq. (7). The derivative of the terms of trade with respect to $L_{F}$ will be (from 7)

$$
\frac{d \frac{P_{x}}{P_{y}}}{d L_{F}}=\frac{\alpha}{\gamma} \frac{h_{x}}{f_{y}} \frac{\bar{L}^{*}-L_{F}^{*}}{\left(\bar{L}-L_{F}\right)^{2}}
$$

As this derivative is positive, higher environmental protection increases the terms of trade in favor of the home country ${ }^{5}$ and thus it rises the welfare of home country and causes a decline in the welfare of foreign country. The lemma is thus proved. Also, note that $\left(d\left(P_{x} / P_{y}\right) / d L_{F}^{*}\right)$ is negative and this shows that the indirect welfare effects of the foreign environmental good production (due to terms of trade changes) are positive for foreign country and negative for home country.

Since terms of trade changes associated with environmental protection generate welfare effects, they also affect the total contribution to global environment by each country. This can be shown more explicitly by considering the case in which the terms of trade are kept constant before and after the environmental policy. As the base case, consider that none of the countries produce environmental good, and thus, the terms of trade will be

$$
\frac{P_{x}}{P_{y}}=\frac{\alpha}{\gamma} \frac{h_{x}}{f_{y}} \frac{\bar{L}^{*}}{\bar{L}}
$$

Now, assume that both countries contribute to environmental protection, but we keep the terms of trade constant at the level in (20). Incorporating this, optimal choices of $L_{F}$ and $L_{F}^{*}$ will be obtained from the solution of

$$
\max _{L_{F}} \frac{\alpha^{\alpha+\gamma}}{(\alpha+\gamma)^{\alpha+\gamma}} \frac{1}{h_{x}^{\alpha} f_{y}^{\gamma}}\left(\frac{\bar{L}^{*}}{\bar{L}}\right)^{\gamma}\left(\bar{L}-L_{F}\right)^{\alpha+\gamma}\left(1+\frac{L_{F}}{h_{f}}+\lambda \frac{L_{F}^{*}}{f_{f}}\right)^{\beta}
$$

and the resulting reaction functions are

$$
L_{F}^{N_{p}}=\frac{\beta}{\alpha+\gamma+\beta} \bar{L}-\frac{\alpha+\gamma}{\alpha+\gamma+\beta} h_{f}-\frac{(\alpha+\gamma) \lambda h_{f}}{(\alpha+\gamma+\beta) f_{f}} L_{F}^{* N_{p}}
$$

where $N_{p}$ in $L_{F}^{N_{p}}$ refers to the non-cooperative contribution when the terms of trade changes are ignored. Similarly

$$
L_{F}^{* N_{p}}=\frac{\beta}{\alpha+\gamma+\beta} \bar{L}^{*}-\frac{\alpha+\gamma}{\alpha+\gamma+\beta} f_{f}-\frac{(\alpha+\gamma) \lambda f_{f}}{(\alpha+\gamma+\beta) h_{f}} L_{F}^{N_{p}}
$$

Now, let us try to compare the total contributions here with the ones obtained above for the case when terms of trade changes were taken into consideration. This is our next result:

Theorem 2 Under the assumptions of Theorem 1, the overall Nash equilibrium contributions of the countries will be under-estimated if the terms of trade changes associated with environmental protection are ignored.

\footnotetext{
${ }^{5}$ Recall that home country exports $X$ and imports $Y$.
} 
Proof By using the symmetry assumption of Theorem 1, we can simplify the reaction functions in (21) and (22) to obtain (note that, in this case $\beta$ may be different from $\alpha$ and $\gamma$ )

$$
L_{\text {Total }}^{N_{p}}=\frac{\beta}{2 \alpha(1+\lambda)+\beta}\left(\bar{L}+\bar{L}^{*}\right)-\frac{4 \alpha}{2 \alpha(1+\lambda)+\beta} h_{f}
$$

and from eqs (9) and (10)

$$
L_{\text {Total }}^{N}=\frac{\beta}{\alpha(1+\lambda)+\beta}\left(\bar{L}+\bar{L}^{*}\right)-\frac{2 \alpha}{\alpha(1+\lambda)+\beta} h_{f}
$$

where $N$ in $L_{F}^{N}$ refers to non-cooperative contribution when terms of trade changes are incorporated as in the previous section.

$$
\text { Since } \frac{\beta}{2 \alpha(1+\lambda)+\beta}<\frac{\beta}{\alpha(1+\lambda)+\beta} \text { and } \frac{4 \alpha}{2 \alpha(1+\lambda)+\beta}>\frac{2 \alpha}{\alpha(1+\lambda)+\beta} \text {, it }
$$
follows from eqs (23) and (24) that $L_{\text {Total }}^{N}>L_{\text {Total }}^{N_{p}}$.

The intuition behind this result is as follows: terms of trade changes affect the marginal product of resources being used in the environmental sector positively; therefore more labor moves to the environmental sector, and so, environmental protection by each country becomes higher when the changes in the prices of private goods are incorporated. This shows that the indirect trade effects of environmental protection are as important as the direct effects. As another important interpretation of Theorem 2, we note that trade connections among countries reduce the free-riding incentives on global environmental protection of others. This enhances the value of trade liberalization, this time, from an environmental perspective.

It is also important to note that the reaction functions described above incorporate a number of opposing effects: (i) transboundary nature of the problem which tends to cause free-riding and thus lowers environmental protection; (ii) trade connections generate terms of trade benefits which tend to increase contribution to environmental protection; (iii) the substitution possibilities between environmental good and private goods tend to lower environmental protection, as lower contribution means higher private income, and so, higher private good consumption. In our case, the last effect is dominant to others and so, reaction functions are always negatively sloped. This also shows the importance of the incorporation of substitution possibilities between private goods and environmental good into the model; most of the other studies in this literature ignore this.

Now, having studied the non-cooperative solutions, the natural next step is to obtain the cooperative solution. How does overall cooperative contribution compare to the non-cooperative one?

\subsection{Cooperative case}

Let us assume that the contributions of each country to global environmental protection are determined by a social planner. The role of the social planner is 
to internalize the production externalities associated with the production of $F$ and $F^{*}$ given the optimal behaviors of the representative consumer in each country. This will be realized through the following maximization problem

$$
\max _{F, F^{*}} U\left(X_{0}, Y_{0}, F, F^{*}\right)+U^{*}\left(X_{0}^{*}, Y_{0}^{*}, F, F^{*}\right)
$$

where $X_{0}, Y_{0}, X_{0}^{*}$, and $Y_{0}^{*}$ are optimal choices of the private goods. When the sum of the welfare levels of the two countries are maximized by the social planner, one may reasonably argue that the solution to the social planner's optimization problem above may result in a lower welfare for one of the countries as compared to its welfare level in the non-cooperative case. Such possibilities must be taken into consideration. The results of this section are independent of such cases, and it can be shown that these important results will hold in cases where both countries obtain higher welfare in the cooperative case as compared to the non-cooperative case.

Replacing $X_{0}, Y_{0}, X_{0}^{*}$, and $Y_{0}^{*}$, by using our results above, the open form of the social planner's problem is

$$
\begin{aligned}
\max _{L_{F}, L_{F}^{*}} \frac{\alpha^{\alpha+\gamma}}{(\alpha+\gamma)^{\alpha+\gamma}} \frac{1}{h_{x}^{\alpha} f_{y}^{\gamma}}\left(\bar{L}^{*}-L_{F}^{*}\right)^{\gamma}\left(\bar{L}-L_{F}\right)^{\alpha}\left(1+\frac{L_{F}}{h_{f}}+\lambda \frac{L_{F}^{*}}{f_{f}}\right)^{\beta} \\
+\frac{\gamma^{\alpha+\gamma}}{(\alpha+\gamma)^{\alpha+\gamma}} \frac{1}{h_{x}^{\alpha} f_{y}^{\gamma}}\left(\bar{L}^{*}-L_{F}^{*}\right)^{\gamma}\left(\bar{L}-L_{F}\right)^{\alpha}\left(1+\frac{L_{F}^{*}}{f_{f}}+\lambda \frac{L_{F}}{h_{f}}\right)^{\beta}
\end{aligned}
$$

To be able to solve the first order conditions of the above maximization problem, we impose that $\beta=1$. Then

$$
L_{F}^{C}=\frac{1}{\alpha+1} \bar{L}-\frac{2 \alpha}{(1+\lambda)(\alpha+1)} h_{f}-\frac{\alpha h_{f}}{(\alpha+1) f_{f}} L_{F}^{* C}
$$

where $C$ in $L_{F}^{C}$ refers to cooperative solution; also, note that

$$
L_{F}^{* C}=\frac{1}{\alpha+1} \bar{L}^{*}-\frac{2 \alpha}{(1+\lambda)(\alpha+1)} f_{f}-\frac{\alpha f_{f}}{(\alpha+1) h_{f}} L_{F}^{C}
$$

The comparison of the overall contribution to global protection in the cooperative case and the one in the non-cooperative case generates our next result:

Theorem 3 Under the assumptions of Theorem 1, if $\beta=1$, the overall contribution of countries to global environmental protection in the cooperative case will be smaller than their contributions in the non-cooperative case when terms of trade changes are taken into consideration.

Proof Using eqs (26) and (27) it can easily be shown that

$$
L_{\text {Total }}^{C}=\frac{1}{2 \alpha+1}\left(\bar{L}+\bar{L}^{*}\right)-\frac{4 \alpha}{2 \alpha(\lambda+1)+\lambda+1} h_{f}
$$

The overall contribution in the non-cooperative case (including terms of trade changes) is given by eq. (24) above. Substitute 1 for $\beta$ in (24). Note that we do 
not need the assumption $\alpha=\gamma=\beta$ here. Since $\frac{1}{\alpha(1+\lambda)+1}>\frac{1}{2 \alpha+1}$ and $\frac{2 \alpha}{\alpha(1+\lambda)+1}<\frac{4 \alpha}{2 \alpha(\lambda+1)+\lambda+1}$, then $L_{\text {Total }}^{N}>L_{\text {Total, }}^{C}$ which is our last theorem.

This result is opposite to the common belief that public goods will be undersupplied in the non-cooperative case as compared to the cooperative case. The main reason for this result is the indirect effects of environmental good production on the welfare levels through changes in the internationally traded goods market. When countries contribute to global environmental protection, they pull away some of their resources from private sector which engages in trade with other countries, and this generates terms of trade effects. If the pattern of specialization remains the same after the pro-environmental policy, the terms of trade of the country producing environmental good improves and this increases the returns to employing labor in the production of environmental good. Thus, more labor moves into the environmental sector as compared to the case when there is no terms of trade effect (or when the terms of trade changes are ignored). In the cooperative case, since the sum of the welfare levels of both countries are maximized, terms of trade effects cancel each other, as an improvement in one country's terms of trade is equivalent to a worsening in the other.

To reinforce this result, we should compare the cooperative result with the non-cooperative result that ignores terms of trade changes. As we show now, the traditional result that the non-cooperative contribution of countries is smaller than their cooperative contributions holds when terms of trade changes are ignored.

Corollary 1 Under the assumptions of Theorem 3, the production of environmental good will be larger in the cooperative case than in the non-cooperative case if the terms of trade changes associated with the production of environmental good are ignored.

Proof Firstly, we replace $\beta$ in (23) by 1 and get the total contribution to environmental protection in the non-cooperative case, in which terms of trade changes are ignored, as

$$
L_{\text {Total }}^{N_{p}}=\frac{1}{2 \alpha(1+\lambda)+1}\left(\bar{L}+\bar{L}^{*}\right)-\frac{4 \alpha}{2 \alpha(1+\lambda)+1} h_{f}
$$

Comparison of $L_{\text {Total }}^{C}$, total cooperative contribution to global protection, in (28) with $L_{\text {Total }}^{N_{p}}$ in (29) clearly shows that $L_{\text {Total }}^{C}>L_{\text {Total }}^{N_{p}}$.

Combining this final result with our previous results, we can state that $L_{\text {Total }}^{N}>L_{\text {Total }}^{C}>L_{\text {Total }}^{N_{p}}$, that is to say, global environmental protection will be higher in the non-cooperative case than in the cooperative case when terms of trade changes are taken into consideration, which is contrary to the conventional results. We get the conventional result, i.e. lower environmental protection in the non-cooperative case, when terms of trade changes are ignored. Thus, we conclude 
that partial equilibrium analysis of global environmental protection which ignores trade connections among countries, may be very misleading.

In an open economy, the public good problem that the agents tend to free-ride on the contribution of others, does not hold when the environmental externality cannot overcome the terms of trade externality associated with the public good production. In a larger picture, it seems possible that, even in a domestic economy, if the contributions of the agents to public good production generate positive side effects for them, then they may also choose to contribute voluntarily; however, since the individuals are most of the time negligible in the total, their decisions will not generally bring about spillovers that may change their incentives to contribute. In our open economy case, however, the countries are not negligible, and the terms of trade benefits arising from environmental protection follow, and through this channel, countries' contributions exceed the optimal contributions associated with the cooperative case, which is a non-conventional result.

\section{Conclusion}

This paper mainly takes up the question whether the suboptimality of the production of a public good in a closed economy extends to open economy case, more explicitly, whether global environmental protection is also subject to free-riding in a model where trade connections among countries are taken into consideration. By studying the case of contributions to global environmental protection in a $2 \times 2$ Ricardian trade model, we demonstrate that there are links between international trade and environmental control, heretofore unappreciated, which might substantially alter the efficacy of various governmental policies to control pollution. One concern about national environmental policies is that, whereas the benefits of certain types of abatement might be international or even worldwide, the costs will be borne strictly by the consumers and firms of the country which institutes the policy. As a result, for those types of pollution which are global (such as greenhouse gases) there will be 'too little' pollution abatement. This pessimistic conclusion might be unwarranted. If the abatement is carried out by diverting resources from export sector, the terms of trade of the country engaged in abatement will improve, with the result that the country is not so reluctant to clean up the environment after all.

It is also demonstrated that there are cases in which contribution to global environmental protection by both countries is the non-cooperative Nash equilibrium of this environmental game. Again contrary to the common belief, the corresponding environmental good production in the non-cooperative case will exceed the one associated with the cooperative solution, under reasonable assumptions. Our results also imply that partial equilibrium studies on free-riding incentives on global protection which ignore the trade connections among countries may very well be misleading. Incorporation of trade connections turns out to be very important as reflected in our results. 


\section{Acknowledgements}

This paper was presented in the second international conference of the European Society for Ecological Economics on Ecological Economics and Development in Geneva, Switzerland, March 1998. I would like to thank Bruce Hamilton, Ali Khan, conference participants and two anonymous reviewers for their valuable comments.

\section{References}

Bac, M. (1996). 'Incomplete Information and Incentives to Free Ride on International Environmental Resources', Journal of Environmental Economics and Management, 30, 30115.

Barrett, S. (1990). 'The Problem of Global Environmental Protection', Oxford Review of Economic Policy, 6, 68-79.

Barrett, S. (1994a). 'Self-Enforcing International Environmental Agreements', Oxford Economic Papers, 46, 878-94.

Barrett, S. (1994b). 'Strategic Environmental Policy and International Trade', Journal of Public Economics, 54, 325-38.

Barrett, S. (1997). 'The Strategy of Trade Sanctions in International Environmental Agreements', Resource and Energy Economics, 19, 345-61.

Bohm, P. (1972). 'Estimating Demand for Public Goods: An Experiment', European Economic Review, 3, 111-30.

Carraro, C. and Siniscalco, D. (1993). 'Strategies for the International Protection of the Environment', Journal of Public Economics, 52, 309-28.

Chichilnisky, G. (1994). 'North-South Trade and the Global Environment', American Economic Review, 84, 851-75.

Daly, H. (1993). 'Debate: Does Free Trade Harm the Environment?', Scientific American, November, 17-29.

Demsetz, H. (1967). 'Toward a Theory of Property Rights', American Economic Review, 57, 347-59.

Heal, G. (1994). 'Formation of International Environmental Agreements', in C. Carraro (ed.), Trade, Innovation, Environment, Kluwer Academic Publishers, Dordrecht.

Kim, O. and Walker, M. (1984). 'The Free Rider Problem: Experimental Evidence', Public Choice, 43, 3-24.

Petrakis, E. and Xepapadeas, A. (1996). 'Environmental Consciousness and Moral Hazard in International Agreements to Protect the Environment', Journal of Public Economics, 60, 95-110.

Piggott, J., Whalley, J., and Wigle, R. (1991). 'International Linkages and Carbon Reduction Initiatives', in K. Anderson and R. Blackhurst (eds), The Greening of World Trade Issues, Harvester Wheatsheaf, London.

Smith, K. and Espinosa, A. (1996). 'Environmental and Trade Policies: Some Methodological Lessons', Discussion Paper No. 96-18, Resources For the Future, Washington, DC.

Smith, V. (1979). 'An Experimental Comparison of Three Public Good Decision Mechanisms', Scandinavian Journal of Economics, 81, 198-215. 
Sweeney, J. (1973). 'An Experimental Investigation of Free Rider Problem', Social Science Research, 2, 227-92.

\section{Appendix}

\section{Proof of Theorem 1}

We can prove this theorem by showing that contributing to global environmental protection is a dominant strategy for both countries. Let us take the home country first. Assume that the foreign country chooses to contribute, then is it the case that the home country will have higher welfare if it also contributes? This requires that the welfare of the home country is greater in Case I (both countries contribute) than in Case IV (foreign country contributes, and home country does not), that is to say

$$
\begin{aligned}
\frac{\alpha^{\alpha+\gamma}}{(\alpha+\gamma)^{\alpha+\gamma}} \frac{1}{h_{x}^{\alpha} f_{y}^{\gamma}}\left(\bar{L}^{*}-L_{F}^{* I}\right)^{\gamma}\left(\bar{L}-L_{F}^{I}\right)^{\alpha}\left(1+\frac{L_{F}^{I}}{h_{f}}+\lambda \frac{L_{F}^{* I}}{f_{f}}\right)^{\beta} & \\
& >\frac{\alpha^{\alpha+\gamma}}{(\alpha+\gamma)^{\alpha+\gamma}} \frac{1}{h_{x}^{\alpha} f_{y}^{\gamma}}\left(\bar{L}^{*}-L_{F}^{* I V}\right)^{\gamma}(\bar{L})^{\alpha}\left(1+\lambda \frac{L_{F}^{* I V}}{f_{f}}\right)^{\beta}
\end{aligned}
$$

where the superscripts $I$ and $I V$ refer to Case I and Case IV, respectively. Making possible cancellations, and noting that $\alpha=\gamma=\beta$, we are left with

$$
\left(\bar{L}^{*}-L_{F}^{* I}\right)\left(\bar{L}-L_{F}^{I}\right)\left(1+\frac{L_{F}^{I}}{h_{f}}+\lambda \frac{L_{F}^{* I}}{f_{f}}\right)>\left(\bar{L}^{*}-L_{F}^{* I V}\right)(\bar{L})\left(1+\lambda \frac{L_{F}^{* I V}}{f_{f}}\right)
$$

From eq. (9), which gives the optimal value of $L_{F}$ as a function of $L_{F}^{*}$, we can show that

$$
L_{F}=\frac{1}{2+\lambda} \bar{L}-\frac{1}{2+\lambda} h_{f}
$$

under symmetry assumptions. Thus, $L_{F}<\bar{L}$, and $L_{F}>0$ if $\bar{L}>h_{f}$; this condition should always be satisfied as $\bar{L}$ represents total labor force and $h_{f}$ is the unit labor requirement in the production of environmental good. So, contribution to global protection $\left(L_{F}\right)$ is feasible and positive. The following useful derivations can easily be done now (note that $L_{F}^{I}=L_{F}^{* I}$ due to symmetry)

$$
\begin{aligned}
\left(1+\frac{L_{F}^{I}}{h_{f}}+\lambda \frac{L_{F}^{* I}}{f_{f}}\right) & =\frac{1+\lambda}{(2+\lambda) h_{f}} \bar{L}+\frac{1}{2+\lambda} \\
\bar{L}-L_{F}^{I} & =\frac{1+\lambda}{2+\lambda} \bar{L}+\frac{1}{2+\lambda} h_{f}
\end{aligned}
$$

and from eq. (10), which gives the value of $L_{F}^{*}$ as a function of $L_{F}$, we get

$$
1+\lambda \frac{L_{F}^{* I V}}{f_{f}}=\frac{\lambda}{2 h_{f}} \bar{L}+\frac{2-\lambda}{2}
$$

by noting that $\beta=\gamma, \bar{L}^{*}=\bar{L}$, and $h_{f}=f_{f}$ (due to symmetry), and that $L_{F}^{I V}=0$ since Case IV refers to no contribution by home country. Thus, (31) simplifies to

$$
\left(\bar{L}^{*}-L_{F}^{* I}\right)\left(\frac{1+\lambda}{2+\lambda} \bar{L}+\frac{1}{2+\lambda} h_{f}\right)\left(\frac{1+\lambda}{(2+\lambda) h_{f}} \bar{L}+\frac{1}{2+\lambda}\right)>\left(\bar{L}^{*}-L_{F}^{* I V}\right) \bar{L}\left(\frac{\lambda}{2 h f} \bar{L}+\frac{2-\lambda}{2}\right)
$$

The first term on the left-hand side is larger than the first term on the right-hand side since $L_{F}^{* I}<L_{F}^{* I V}$ (see eq. (10) with $L_{F}^{I V}=0$ and $L_{F}^{I}>0$ ). The third term on the LHS is larger than the second term on the RHS if $h_{f}<(1+\lambda) /(2+\lambda)$, which is assumed to hold in Theorem 1. Finally, Theorem 1 assumes that the second term on the LHS is larger than the 
last term on the RHS, i.e., $(1+\lambda) /(2+\lambda) \bar{L}+(1 /(2+\lambda)) h_{f}>\left(\lambda / 2 h_{f}\right) \bar{L}+(2-\lambda) / 2$. Thus, LHS will be larger than RHS in eq. (30) and so, the home country will choose to contribute.

Now, we need to show that contribution will again be dominant for the home country when the foreign country does not contribute. This requires that the utility of the home country is greater in Case III (home country contributes, and foreign country does not) than in Case II (none contributes), i.e.

$$
\frac{\alpha^{\alpha+\gamma}}{(\alpha+\gamma)^{\alpha+\gamma}} \frac{1}{h_{x}^{\alpha} f_{y}^{\gamma}} \bar{L}^{* \gamma}\left(\bar{L}-L_{F}\right)^{\alpha}\left(1+\frac{L_{F}}{h_{f}}\right)^{\beta}>\frac{\alpha^{\alpha+\gamma}}{(\alpha+\gamma)^{\alpha+\gamma}} \frac{1}{h_{x}^{\alpha} f_{y}^{\gamma}} \bar{L}^{* \gamma} \bar{L}^{\alpha}
$$

which, under symmetry assumptions, simplifies to

$$
\left(\bar{L}-L_{F}\right)\left(1+\frac{L_{F}}{h_{f}}\right)>\bar{L}
$$

The left-hand side of (33) represents no contribution by foreign country, i.e., $L_{F}^{*}=0$. Putting this in the optimal value of $L_{F}$ in (9) and using $\beta=\alpha$, we get

Substituting these in eq. (33)

$$
\begin{aligned}
\bar{L}-L_{F} & =\frac{1}{2} \bar{L}+\frac{1}{2} h_{f} \\
1+\frac{L_{F}}{h_{f}} & =\frac{1}{2} \frac{\bar{L}}{h_{f}}+\frac{1}{2}
\end{aligned}
$$

which simplifies to

$$
\frac{1}{4 h_{f}}\left(\bar{L}+h_{f}\right)^{2}>\bar{L}
$$

$$
\left(\bar{L}-h_{f}\right)^{2}>0
$$

which is always true. Again contribution is dominant strategy for home country.

Since countries are symmetric, contribution to global protection will be dominant strategy for foreign country too. Thus, in the Nash equilibrium both countries will choose to contribute to global environmental protection.

\section{A brief note on the derivation of reaction functions in the cooperative case}

The maximization problem given in eq. (25) results in the following first order conditions with respect to $L_{F}$ of home country

$$
\begin{aligned}
& A\left(\bar{L}^{*}-L_{F}^{*}\right)^{\gamma}\left\{-\alpha\left(\bar{L}-L_{F}\right)^{\alpha-1}\left(1+\frac{L_{F}}{h_{f}}+\lambda \frac{L_{F}^{*}}{f_{f}}\right)^{\beta}+\frac{\beta}{h_{f}}\left(\bar{L}-L_{F}\right)^{\alpha}\left(1+\frac{L_{F}}{h_{f}}+\lambda \frac{L_{F}^{*}}{f_{f}}\right)^{\beta-1}\right\} \\
& +B\left(\bar{L}^{*}-L_{F}^{*}\right)^{\gamma}\left\{-\alpha\left(\bar{L}-L_{F}\right)^{\alpha-1}\left(1+\lambda \frac{L_{F}}{h_{f}}+\frac{L_{F}^{*}}{f_{f}}\right)^{\beta}+\frac{\lambda \beta}{h_{f}}\left(\bar{L}-L_{F}\right)^{\alpha}\left(1+\lambda \frac{L_{F}}{h_{f}}+\frac{L_{F}^{*}}{f_{f}}\right)^{\beta-1}\right\}=0
\end{aligned}
$$
where $A=\left(\alpha^{\alpha+\gamma} /(\alpha+\gamma)^{\alpha+\gamma}\right)\left(1 / h_{x}^{\alpha} f_{y}^{\gamma}\right)$ and $B=\left(\gamma^{\alpha+\gamma} /(\alpha+\gamma)^{\alpha+\gamma}\right)\left(1 / h_{x}^{\alpha} f_{y}^{\gamma}\right)$.

A similar expression will result when derivative of $(25)$ is taken with respect to $L_{F}^{*}$. As seen clearly, it is difficult to solve for $L_{F}$ in the above expression; however, if we let $\beta=1$ as in Theorem 3, we will be able to solve for reaction functions under the usual symmetry assumptions. The resulting reaction functions are given in eqs (26) and (27) in Section 3. 